\title{
BAD BOUNDARY BEHAVIOR IN STAR INVARIANT SUBSPACES II
}

\author{
Andreas Hartmann and William T. Ross \\ Université de Bordeaux, Institut de Mathématiques de Bordeaux \\ 351 cours de la Libération, 33405 Talence, France; hartmann@math.u-bordeaux.fr \\ University of Richmond, Department of Mathematics and Computer Science \\ 212 Jepson Hall, 28 Westhampton Way, VA 23173, U.S.A.; wross@richmond.edu
}

\begin{abstract}
We continue our study begun in [HR11] concerning the radial growth of functions in the model spaces $\left(I H^{2}\right)^{\perp}$.
\end{abstract}

\section{Introduction}

Suppose $I=B S_{\mu}$ is an inner function with Blaschke factor $B$, with zeros $\left\{\lambda_{n}\right\}_{n \geq 1}$ in the open unit disk $\mathbf{D}$ repeated according to multiplicity, and singular inner factor $S_{\mu}$ with associated positive singular measure $\mu$ on the unit circle $\mathbf{T}$. The following result was shown by Frostman in 1942 for Blaschke products (see [Fro42] or [CL66]) and by Ahern-Clark for general inner functions [AC71, Lemma 3].

Theorem 1.1. (Frostman, 1942; Ahern-Clark, 1971) Let $\zeta \in \mathbf{T}$ and $I$ be inner with $\mu(\{\zeta\})=0$. Then the following assertions are equivalent.

(1) Every divisor of $I$ has a radial limit of modulus one at $\zeta$.

(2) Every divisor of $I$ has a radial limit at $\zeta$.

(3) The following condition holds

$$
\sum_{n \geq 1} \frac{1-\left|\lambda_{n}\right|}{\left|\zeta-\lambda_{n}\right|}+\int_{\mathbf{T}} \frac{1}{\left|\zeta-e^{i t}\right|} d \mu\left(e^{i t}\right)<\infty .
$$

Based on a stronger condition than the above, Ahern and Clark [AC70] were able to characterize "good" non-tangential boundary behavior of functions in the model spaces $\left(I H^{2}\right)^{\perp}$ of the classical Hardy space $H^{2}$ (see [Nik86] for a very complete treatment of model spaces).

Theorem 1.3. [AC70] Let $I=B S_{\mu}$ be an inner function with zeros $\left\{\lambda_{n}\right\}_{n \geq 1}$ and associated singular measure $\mu$. For $\zeta \in \mathbf{T}$, the following are equivalent:

(1) Every $f \in\left(I H^{2}\right)^{\perp}$ has a radial limit at $\zeta$.

(2) The following condition holds

$$
\sum_{n \geq 1} \frac{1-\left|\lambda_{n}\right|}{\left|\zeta-\lambda_{n}\right|^{2}}+\int_{\mathbf{T}} \frac{1}{\left|\zeta-e^{i t}\right|^{2}} d \mu\left(e^{i t}\right)<\infty .
$$

doi:10.5186/aasfm.2012.3744

2010 Mathematics Subject Classification: Primary 30J10, 30A12, 30A08.

Key words: Hardy spaces, star invariant subspaces, non-tangential limits, inner functions, unconditional sequences, generalized Carleson condition.

First named author partially supported by ANR project FRAB, ANR-09-BLAN-0058-02. 
In this paper, we will study what happens when we are somewhere in between the Frostman condition (1.2) and the Ahern-Clark condition (1.4). In order to do so we will introduce an auxiliary function. Let $\varphi:(0,2] \rightarrow \mathbf{R}^{+}$be a positive increasing function such that

(1) $x \rightarrow \frac{\varphi(x)}{x}$ is bounded,

(2) $x \longmapsto \frac{\varphi(x)}{x^{2}}$ is decreasing,

(3) $\varphi(x) \asymp \varphi(x+o(x)), x \downarrow 0$.

Such a function $\varphi$ will be called admissible. One can check that functions such as $\varphi(x)=x^{p}, 1 \leq p \leq 2$, and $\varphi(x)=x^{p} \log (1 / x), 1<p<2$, are admissible. Our main result is the following.

Theorem 1.5. Let $I=B S_{\mu}$ be an inner function with zeros $\left\{\lambda_{n}\right\}_{n \geq 1}$ and associated singular measure $\mu, \varphi$ an admissible function, and $\zeta \in \mathbf{T}$. If

$$
\sum_{n \geq 1} \frac{1-\left|\lambda_{n}\right|}{\varphi\left(\left|\zeta-\lambda_{n}\right|\right)}+\int_{\mathbf{T}} \frac{1}{\varphi\left(\left|\zeta-e^{i t}\right|\right)} d \mu\left(e^{i t}\right)<\infty
$$

then every $f \in\left(I H^{2}\right)^{\perp}$ satisfies

$$
|f(r \zeta)| \lesssim \frac{\sqrt{\varphi(1-r)}}{1-r}
$$

When $\varphi(x)=x$ then we are in the Frostman situation (1.2) and no restriction is given for the growth of $f$ since generic functions in $H^{2}$ satisfy the growth condition

$$
|f(r \zeta)|=o\left(\frac{1}{\sqrt{1-r}}\right)
$$

On the other hand, when $\varphi(x)=x^{2}$ we reach the Ahern-Clark situation (1.4). For other $\varphi$ such as $\varphi(x)=x^{3 / 2}$ or perhaps $\varphi(x)=x^{2} / \log (e / x)$ we get that even though functions in $\left(I H^{2}\right)^{\perp}$ can be poorly behaved (as in the title of this paper), the growth is controlled.

There is some history behind these types of problems. When $\varphi(x)=x^{2 N+2}$, where $N=0,1,2, \cdots$, Ahern and Clark [AC70] showed that (1.6) is equivalent to the condition that $f^{(j)}, 0 \leq j \leq N$, have radial limits at $\zeta$ for every $f \in\left(I H^{2}\right)^{\perp}$. When $\varphi(x)=x^{p}, p \in(1, \infty)$, Cohn [Coh86] showed that (1.6) is equivalent to the condition that every $f \in H^{q} \cap I \overline{H_{0}^{q}}$, where $q=p(p-1)^{-1}$, has a finite radial limit at $\zeta$.

Why did we write this second paper? In [HR11] we discussed controlled growth of functions from $\left(B H^{2}\right)^{\perp}$, where $B$ is a Blaschke product not satisfying the condition (1.4) of the Ahern-Clark theorem. We have a general result but stated in very different terms, and using very different techniques, than the paper here. In particular, in [HR11] we obtain two-sided estimates for the reproducing kernels which yields more precise results. The results presented here are one-sided estimates but are for general inner functions and not just Blaschke products. 


\section{Proof of the main result}

It is well known that $\left(I H^{2}\right)^{\perp}$ is a reproducing kernel Hilbert space with kernel function

$$
k_{\lambda}^{I}(z):=\frac{1-\overline{I(\lambda)} I(z)}{1-\bar{\lambda} z} .
$$

It suffices to prove Theorem 1.5 for $\zeta=1$. If $\|\cdot\|$ denotes the norm in $H^{2}$, the estimate in (1.7) follows from the following result along with the obvious estimate

$$
|f(r)| \leq\|f\|\left\|k_{r}^{I}\right\|, \quad f \in\left(I H^{2}\right)^{\perp}, \quad r \in(0,1) .
$$

Theorem 2.1. Let $I=B S_{\mu}$ be an inner function with zeros $\left\{\lambda_{n}\right\}_{n \geq 1}$ and associated singular measure $\mu$ and $\varphi$ be an admissible function. If

$$
\sum_{n \geq 1} \frac{1-\left|\lambda_{n}\right|}{\varphi\left(\left|1-\lambda_{n}\right|\right)}+\int_{\mathbf{T}} \frac{1}{\varphi\left(\left|1-e^{i t}\right|\right)} d \mu\left(e^{i t}\right)<\infty,
$$

then

$$
\left\|k_{r}^{I}\right\|^{2} \lesssim \frac{\varphi(1-r)}{(1-r)^{2}}
$$

Proof. Our first observation is that since $x \longmapsto \varphi(x) / x$ is bounded, (2.2) implies condition (1.2). By Theorem 1.1 this implies that $\lim _{r \rightarrow 1^{-}}|B(r)|=\lim _{r \rightarrow 1^{-}}\left|S_{\mu}(r)\right|=$ 1. Hence

$$
\left\|k_{r}^{I}\right\|^{2}=\frac{1-|I(r)|^{2}}{1-r^{2}}=\frac{1-\exp \left(\log \left(|I(r)|^{2}\right)\right)}{1-r^{2}}=\frac{1-\exp \left(\log \left(|B(r)|^{2}+\log \left|S_{\mu}(r)\right|^{2}\right)\right)}{1-r^{2}},
$$

and since $\log |B(r)| \rightarrow 0$ and $\log \left|S_{\mu}(r)\right| \rightarrow 0$ when $r \rightarrow 1$, we get

$$
\begin{aligned}
\left\|k_{r}^{I}\right\|^{2} & =\frac{1-\exp \left(\log |B(r)|^{2}+\log \left|S_{\mu}(r)\right|^{2}\right)}{1-r^{2}} \\
& =\frac{1-\left(1+\left(\log |B(r)|^{2}+\log \left|S_{\mu}(r)\right|^{2}\right)+o\left(\log |B(r)|^{2}+\log \left|S_{\mu}(r)\right|^{2}\right)\right)}{1-r^{2}} \\
& \sim \frac{\log |B(r)|^{-2}+\log \left|S_{\mu}(r)\right|^{-2}}{1-r^{2}} .
\end{aligned}
$$

Thus to prove the estimate in (2.3) we need to prove

$$
\frac{\log |B(r)|^{-2}}{1-r^{2}} \lesssim \frac{\varphi(1-r)}{(1-r)^{2}}
$$

and

$$
\frac{\log \left|S_{\mu}(r)\right|^{-2}}{1-r^{2}} \lesssim \frac{\varphi(1-r)}{(1-r)^{2}}
$$

Case 1: The Blaschke product $B$. First note that from the Frostman condition (1.2) we get

$$
\frac{1-\left|\lambda_{n}\right|}{\left|1-\lambda_{n}\right|} \longrightarrow 0
$$

This condition implies that for every Stolz angle at $1, \Gamma_{\alpha}:=\{z \in \mathbf{D}:|1-z| \leq$ $\alpha(1-|z|)\}$, where $\alpha>1$, there is an index $n_{0}$ such that for $n \geq n_{0}$ the points $\lambda_{n}$ are outside $\Gamma_{\alpha}$, implying that $\left\{\lambda_{n}\right\}_{n \geq 1}$ goes tangentially to 1 . In particuliar, for $n \geq n_{0}$, 
$\lambda_{n}$ will be pseudohyperbolically far from the radius $[0,1)$, i.e., there is a $\delta$ such that for every $n \geq n_{0}$ and $r \in[0,1)$,

$$
\left|b_{\lambda_{n}}(r)\right| \geq \delta
$$

Here $b_{\lambda}=(\lambda-z) /(1-\bar{\lambda} z)$ is the usual Blaschke factor and $\rho(\lambda, z)=\left|b_{\lambda}(z)\right|$ defines the pseudohyperbolic distance between $\lambda$ and $z$. This implies

$$
\log \frac{1}{\left|b_{\lambda_{n}}(r)\right|^{2}} \asymp 1-\left|b_{\lambda_{n}}(r)\right|^{2} .
$$

It is well known that

$$
1-\left|b_{\lambda_{n}}(r)\right|^{2}=\frac{\left(1-r^{2}\right)\left(1-\left|\lambda_{n}\right|^{2}\right)}{\left|1-r \overline{\lambda_{n}}\right|^{2}} .
$$

Thus

$$
\frac{\log |B(r)|^{-2}}{1-r^{2}}=\frac{1}{1-r^{2}} \sum_{n \geq 1} \log \frac{1}{\left|b_{\lambda_{n}}(r)\right|^{2}} \asymp \sum_{n \geq 1} \frac{1-\left|\lambda_{n}\right|^{2}}{\left|1-\overline{\lambda_{n}} r\right|^{2}} .
$$

Now let $\lambda_{n}=r_{n} e^{i \theta_{n}}$. We need the following two easy estimates:

$$
\begin{aligned}
\left|1-\rho e^{i \theta}\right|^{2} & \asymp(1-\rho)^{2}+\theta^{2}, \quad \rho \approx 1, \theta \approx 0, \\
\left(|z|^{2}+|w|^{2}\right)^{1 / 2} & \asymp|z|+|w|, \quad z, w \in \mathbf{C} .
\end{aligned}
$$

In particular, $\left|1-\lambda_{n}\right|^{2} \asymp\left(1-r_{n}\right)^{2}+\theta_{n}^{2}$. We now remember condition (2.6) which implies that $1-r_{n}=1-\left|\lambda_{n}\right|=o\left(\left|1-\lambda_{n}\right|\right)=o\left(\left(1-r_{n}\right)+\theta_{n}\right)$ so that necessarily $1-r_{n}=o\left(\theta_{n}\right)$. Hence

$$
\left|1-\bar{\lambda}_{n} r\right|^{2} \asymp\left(1-r_{n} r\right)^{2}+\theta_{n}^{2}=\left(1-r_{n}+r_{n}(1-r)\right)^{2}+\theta_{n}^{2} \asymp(1-r)^{2}+\theta_{n}^{2} .
$$

The estimate in (2.7) yields

$$
\begin{aligned}
\frac{\log |B(r)|^{-2}}{1-r^{2}} & \asymp \sum_{n \geq 1} \frac{1-\left|\lambda_{n}\right|^{2}}{\left|1-\overline{\lambda_{n}} r\right|^{2}} \asymp \sum_{n \geq 1} \frac{1-r_{n}}{(1-r)^{2}+\theta_{n}^{2}} \\
& \asymp \sum_{\left\{n: 1-r<\theta_{n}\right\}} \frac{1-r_{n}}{\theta_{n}^{2}}+\sum_{\left\{n: 1-r \geq \theta_{n}\right\}} \frac{1-r_{n}}{(1-r)^{2}} \\
& =\sum_{\left\{n: 1-r<\theta_{n}\right\}} \frac{1-r_{n}}{\theta_{n}^{2}}+\frac{1}{(1-r)^{2}} \sum_{\left\{n: 1-r \geq \theta_{n}\right\}}\left(1-r_{n}\right) .
\end{aligned}
$$

Let us discuss each summand in (2.10) individually. For the first, we use the fact that $\varphi$ is admissible and so $\varphi(\theta) \asymp \varphi\left(\left|1-e^{i \theta}\right|\right)$ to get

$$
\begin{aligned}
\sum_{\left\{n: 1-r<\theta_{n}\right\}} \frac{1-r_{n}}{\theta_{n}^{2}} & =\sum_{\left\{n: 1-r<\theta_{n}\right\}} \frac{1-r_{n}}{\sqrt{\varphi\left(\theta_{n}\right)} \theta_{n}^{2} / \sqrt{\varphi\left(\theta_{n}\right)}} \\
& \leq \underbrace{\left(\sum_{\left\{n: 1-r<\theta_{n}\right\}} \frac{1-r_{n}}{\varphi\left(\theta_{n}\right)}\right)^{1 / 2}\left(\sum_{\left\{n: 1-r<\theta_{n}\right\}} \frac{1-r_{n}}{\theta_{n}^{4} / \varphi\left(\theta_{n}\right)}\right)^{1 / 2}}_{\text {bounded by assumption }} \\
& \lesssim\left(\sum_{\left\{n: 1-r<\theta_{n}\right\}} \frac{1-r_{n}}{\varphi\left(\theta_{n}\right)\left(\theta_{n}^{2} / \varphi\left(\theta_{n}\right)\right)^{2}}\right)^{1 / 2} .
\end{aligned}
$$


Since $\varphi$ is admissible, $x \rightarrow \varphi(x) / x^{2}$ is decreasing. Hence we can bound $\theta_{n}^{2} / \varphi\left(\theta_{n}\right)$ below in this last sum by $(1-r)^{2} / \varphi(1-r)$. This together with $(2.2)$ gives us

$$
\sum_{\left\{n: 1-r<\theta_{n}\right\}} \frac{1-r_{n}}{\theta_{n}^{2}} \lesssim \frac{\varphi(1-r)}{(1-r)^{2}}\left(\sum_{\left\{n: 1-r<\theta_{n}\right\}} \frac{1-r_{n}}{\varphi\left(\theta_{n}\right)}\right)^{1 / 2} \lesssim \frac{\varphi(1-r)}{(1-r)^{2}} .
$$

For the second sum in (2.10) we have

$$
\begin{aligned}
\sum_{\left\{n: 1-r \geq \theta_{n}\right\}}\left(1-r_{n}\right) & =\sum_{\left\{n: 1-r \geq \theta_{n}\right\}}\left(1-r_{n}\right) \frac{\sqrt{\varphi\left(\theta_{n}\right)}}{\sqrt{\varphi\left(\theta_{n}\right)}} \\
& \leq \underbrace{\left(\sum_{\left\{n: 1-r \geq \theta_{n}\right\}} \frac{\left(1-r_{n}\right)}{\varphi\left(\theta_{n}\right)}\right)^{1 / 2}}_{\text {bounded by assumption }}\left(\sum_{\left\{n: 1-r \geq \theta_{n}\right\}}\left(1-r_{n}\right) \varphi\left(\theta_{n}\right)\right)^{1 / 2} \\
& \lesssim \sqrt{\varphi(1-r)}\left(\sum_{\left\{n: 1-r \geq \theta_{n}\right\}}\left(1-r_{n}\right)\right)^{1 / 2},
\end{aligned}
$$

where we have used the fact that $\varphi$ is increasing. Dividing through the square root of the sum in this last inequality (and then squaring) implies

$$
\sum_{\left\{n: 1-r \geq \theta_{n}\right\}}\left(1-r_{n}\right) \lesssim \varphi(1-r)
$$

This verifies (2.4).

Case 2: The singular inner factor $S_{\mu}$. This case is very similar to the first case. Indeed,

$$
\frac{\log \left|S_{\mu}(r)\right|^{-2}}{1-r^{2}}=2 \int_{\mathbf{T}} \frac{1}{\left|1-r e^{i \theta}\right|^{2}} d \mu\left(e^{i \theta}\right) \asymp \int_{\mathbf{T}} \frac{1}{(1-r)^{2}+\theta^{2}} d \mu\left(e^{i \theta}\right),
$$

where we have again used (2.8). As in the Blaschke situation we split the integral into two parts depending on which term in the denominator dominates:

$$
\begin{aligned}
\frac{\log \left|S_{\mu}(r)\right|^{-2}}{1-r^{2}} & \lesssim \int_{\{\theta: 1-r \leq \theta\}} \frac{1}{(1-r)^{2}+\theta^{2}} d \mu\left(e^{i \theta}\right)+\int_{\{\theta: 1-r \geq \theta\}} \frac{1}{(1-r)^{2}+\theta^{2}} d \mu\left(e^{i \theta}\right) \\
& \asymp \int_{\{\theta: 1-r \leq \theta\}} \frac{1}{\theta^{2}} d \mu\left(e^{i \theta}\right)+\frac{1}{(1-r)^{2}} \int_{\{\theta: 1-r \geq \theta\}} d \mu\left(e^{i \theta}\right) .
\end{aligned}
$$

Let us consider the first integral:

$$
\begin{aligned}
\int_{\{\theta: 1-r \leq \theta\}} \frac{1}{\theta^{2}} d \mu\left(e^{i \theta}\right) & =\int_{\{\theta: 1-r \leq \theta\}} \frac{1}{\sqrt{\varphi(\theta)} \theta^{2} / \sqrt{\varphi(\theta)}} d \mu\left(e^{i \theta}\right) \\
& \leq\left(\int_{\{\theta: 1-r \leq \theta\}} \frac{1}{\varphi(\theta)} d \mu\left(e^{i \theta}\right)\right)^{1 / 2}\left(\int_{\{\theta: 1-r \leq \theta\}} \frac{1}{\theta^{4} / \varphi(\theta)} d \mu\left(e^{i \theta}\right)\right)^{1 / 2} .
\end{aligned}
$$

Again, $\left|1-e^{i \theta}\right| \asymp \theta$. Then using the hypothesis of admissibility we have $\varphi(\theta) \asymp$ $\varphi\left(\left|1-e^{i \theta}\right|\right)$ and so

$$
\int \frac{1}{\varphi(\theta)} d \mu\left(e^{i \theta}\right) \asymp \int \frac{1}{\varphi\left(\left|1-e^{i \theta}\right|\right)} d \mu\left(e^{i \theta}\right)
$$


which is bounded by assumption. Hence,

$$
\begin{aligned}
\int_{\{\theta: 1-r \leq \theta\}} \frac{1}{\theta^{2}} d \mu\left(e^{i \theta}\right) & \lesssim\left(\int_{\{\theta: 1-r \leq \theta\}} \frac{1}{\theta^{4} / \varphi(\theta)} d \mu\left(e^{i \theta}\right)\right)^{1 / 2} \\
& =\left(\int_{\{\theta: 1-r \leq \theta\}} \frac{\varphi^{2}(\theta)}{\varphi(\theta) \theta^{4}} d \mu\left(e^{i \theta}\right)\right)^{1 / 2} .
\end{aligned}
$$

Now using the fact that $x \longrightarrow \varphi(x) / x^{2}$ is decreasing we obtain

$$
\varphi^{2}(\theta) / \theta^{4} \leq(\varphi(1-r))^{2} /(1-r)^{4}
$$

and

$$
\int_{\{\theta: 1-r \leq \theta\}} \frac{1}{\theta^{2}} d \mu\left(e^{i \theta}\right) \lesssim \frac{\varphi(1-r)}{(1-r)^{2}}\left(\int_{\{\theta: 1-r \leq \theta\}} \frac{1}{\varphi(\theta)} d \mu\left(e^{i \theta}\right)\right)^{1 / 2} \lesssim \frac{\varphi(1-r)}{(1-r)^{2}} .
$$

We turn to the second integral in (2.11) to get

$$
\begin{aligned}
\int_{\{\theta: 1-r \geq \theta\}} d \mu\left(e^{i \theta}\right) & =\int_{\{\theta: 1-r \geq \theta\}} \frac{\sqrt{\varphi(\theta)}}{\sqrt{\varphi(\theta)}} d \mu\left(e^{i \theta}\right) \\
& \leq\left(\int_{\{\theta: 1-r \geq \theta\}} \varphi(\theta) d \mu\left(e^{i \theta}\right)\right)^{1 / 2}\left(\int_{\{\theta: 1-r \geq \theta\}} \frac{1}{\varphi(\theta)} d \mu\left(e^{i \theta}\right)\right)^{1 / 2} .
\end{aligned}
$$

We have already seen above that the second factor is bounded by assumption. Using the fact that $\varphi$ is increasing we get

$$
\int_{\{\theta: 1-r \geq \theta\}} d \mu\left(e^{i \theta}\right) \lesssim\left(\int_{\{\theta: 1-r \geq \theta\}} \varphi(\theta) d \mu\left(e^{i \theta}\right)\right)^{1 / 2} \leq \sqrt{\varphi(1-r)}\left(\int_{\{\theta: 1-r \geq \theta\}} d \mu\left(e^{i \theta}\right)\right)^{1 / 2} .
$$

Dividing through by the integral (and then squaring), we obtain

$$
\int_{\{\theta: 1-r \geq \theta\}} d \mu\left(e^{i \theta}\right) \lesssim \varphi(1-r)
$$

which verifies (2.5).

\section{An example}

The Blaschke situation was discussed in [HR11] where we obtained two-sided estimates for the reproducing kernels. It can be shown with concrete examples that the estimates from Theorem 2.1 are in general weaker than those obtained in [HR11] for Blaschke products.

Let us discuss the simplest case, in fact close enough to a Blaschke product, that a singular inner function $S_{\mu}$ with a discrete measure $\mu$. Let

$$
\mu=\sum_{n \geq 1} \alpha_{n} \delta_{\zeta_{n}}
$$

where $\delta_{\zeta_{n}} \in \mathbf{T}$ and $\alpha_{n}$ are positive numbers with $\sum_{n} \alpha_{n}<\infty$ guaranteeing that $\mu$ is a finite measure on $\mathbf{T}$. Let us fix

$$
\zeta_{n}=e^{i \theta_{n}}=e^{i / 2^{n}}, \quad \alpha_{n}=\left(\frac{1}{2^{\varepsilon}}\right)^{n}, \quad n=1,2, \ldots .
$$


Also let $\varphi(t)=t^{\gamma}$ which defines an admissible function for $1<\gamma<2$. In order to have condition (2.2) it is necessary and sufficient to have

$$
\sum_{n} \alpha_{n} \frac{1}{\varphi\left(\left|1-e^{i \theta_{n}}\right|\right)} \simeq \sum_{n} \frac{1}{2^{n \varepsilon}} \frac{1}{\varphi\left(1 / 2^{n}\right)} \simeq \sum_{n} 2^{(\gamma-\varepsilon) n}<\infty
$$

which is equivalent to $\gamma<\varepsilon$. We suppose that

$$
\gamma<\varepsilon<2
$$

By Theorem 2.1 we deduce that

$$
\left\|k_{r}^{I}\right\|^{2} \lesssim \frac{\varphi(1-r)}{(1-r)^{2}}=\left(\frac{1}{1-r}\right)^{2-\gamma},
$$

and hence

$$
|f(r)| \lesssim \frac{1}{(1-r)^{1-\gamma / 2}}, \quad f \in\left(S_{\mu} H^{2}\right)^{\perp},
$$

which is slower growth than the standard estimate

$$
|f(r)| \lesssim \frac{1}{(1-r)^{1 / 2}}, \quad f \in H^{2} .
$$

In this situation, it is actually possible to get a double-sided estimate for the reproducing kernel: since $\varphi$ is admissible, Theorem 1.1 implies that $I(r) \longrightarrow \eta \in \mathbf{T}$ when $r \rightarrow 1^{-}$. In particular for $r \in(0,1)$, this implies that

$$
|I(r)|=\exp \left(-\sum_{n} \alpha_{n} \frac{1-r^{2}}{\left|\zeta_{n}-r\right|^{2}}\right) \sim 1-\sum_{n} \alpha_{n} \frac{1-r^{2}}{\left|\zeta_{n}-r\right|^{2}} .
$$

Let us consider the reproducing kernel of $\left(S_{\mu} H^{2}\right)^{\perp}$ at $r=\rho_{N}=1-2^{-N}$. Indeed,

$$
\left\|k_{\rho_{N}}^{I}\right\|^{2}=\frac{1-\left|I\left(\rho_{N}\right)\right|^{2}}{1-\rho_{N}^{2}} \asymp \sum_{n} \frac{\alpha_{n}}{\left|\zeta_{n}-\rho_{N}\right|^{2}} .
$$

Now using (2.8)

$$
\left|\zeta_{n}-\rho_{N}\right|^{2} \asymp \frac{1}{2^{2 n}}+\frac{1}{2^{2 N}}
$$

and so

$$
\begin{aligned}
\left\|k_{\rho_{N}}^{I}\right\|^{2} & \asymp \sum_{n} \frac{\alpha_{n}}{1 / 2^{2 n}+1 / 2^{2 N}}=\sum_{n \leq N} \frac{\alpha_{n}}{1 / 2^{2 n}}+\sum_{n>N} \frac{\alpha_{n}}{1 / 2^{2 N}} \\
& \asymp \sum_{n \leq N} 2^{(2-\varepsilon) n}+2^{2 N} \sum_{n>N} \frac{1}{2^{\varepsilon n}} \asymp 2^{(2-\varepsilon) N}=\left(\frac{1}{1-\rho_{N}}\right)^{2-\varepsilon}
\end{aligned}
$$

or, equivalently,

$$
\left\|k_{\rho_{N}}^{I}\right\| \asymp\left(\frac{1}{1-\rho_{N}}\right)^{1-\varepsilon / 2}
$$

(the estimate extends to the whole radius). As a consequence, the estimate from Theorem 2.1 is not optimal, though it is possible to come closer to it by choosing e.g., $\varphi(t)=t^{\varepsilon} / \log ^{1+\gamma}(1 / t), \gamma>0$. 


\section{A lower estimate}

We finish this paper with a construction of an $f \in\left(S_{\mu} H^{2}\right)^{\perp}$, with $\mu$ the discrete measure discussed in the previous section, getting close to the growth given by the norm of the reproducing kernels thoughout a whole Stolz angle at 1. As in [HR11] our construction will be based on unconditional sequences. We need to recall some material on generalized interpolation in Hardy spaces for which we refer the reader to [Nik02, Section C3]. Let $I=\prod_{n} I_{n}$ be a factorization of an inner function $I$ into inner functions $I_{n}, n \in \mathbf{N}$. The sequence $\left\{I_{n}\right\}_{n \geq 1}$ satisfies the generalized Carleson condition, sometimes called the Carleson-Vasyunin condition, which we will write $\left\{I_{n}\right\}_{n \geq 1} \in(C V)$, if there is a $\delta>0$ such that

$$
|I(z)| \geq \delta \inf _{n \geq 1}\left|I_{n}(z)\right|, \quad z \in \mathbf{D} .
$$

In the special case of a Blaschke product $B=B_{\Lambda}$ with simple zeros $\Lambda=\left\{\lambda_{n}\right\}_{n \geq 1}$ and $I_{n}=b_{\lambda_{n}}$, this is equivalent to the well-known Carleson condition $\inf _{n}\left|B_{\Lambda \backslash\left\{\lambda_{n}\right\}}\left(\bar{\lambda}_{n}\right)\right| \geq$ $\delta>0$.

If $\left\{I_{n}\right\}_{n \geq 1} \in(C V)$ then $\left\{\left(I_{n} H^{2}\right)^{\perp}\right\}_{n \geq 1}$ is an unconditional basis for $\left(I H^{2}\right)^{\perp}$ meaning that every $f \in\left(I H^{2}\right)^{\perp}$ can be written uniquely as

$$
f=\sum_{n \geq 1} f_{n}, \quad f_{n} \in\left(I_{n} H^{2}\right)^{\perp},
$$

with

$$
\|f\|^{2} \asymp \sum_{n \geq 1}\left\|f_{n}\right\|^{2} .
$$

In our situation we have $I=S_{\mu}$ and

$$
I_{n}=e^{\alpha_{n} \frac{z+\zeta_{n}}{z-\zeta_{n}}} .
$$

The corresponding spaces $\left(I_{n} H^{2}\right)^{\perp}$ are known to be isometrically isomorphic to the Paley-Wiener space of analytic functions of exponential type $\alpha_{n} / 2$ and square integrable on the real axis. In this situation, a sufficient condition for (4.1) is known:

$$
\sup _{n \geq 1} \sum_{k \neq n} \frac{\mu\left(\left\{\zeta_{n}\right\}\right) \mu\left(\left\{\zeta_{k}\right\}\right)}{\left|\zeta_{n}-\zeta_{k}\right|^{2}}<\infty
$$

(see [Nik86, Corollary 6, p. 247]). So, since $\varepsilon>1$ by (3.1), we have

$$
\begin{aligned}
\sum_{k \neq n} \frac{1 / 2^{\varepsilon n} 1 / 2^{\varepsilon k}}{\left|1 / 2^{n}-1 / 2^{k}\right|^{2}} & \simeq 1 / 2^{\varepsilon n} \sum_{k<n} \frac{1 / 2^{\varepsilon k}}{\left|1 / 2^{k}\right|^{2}}+1 / 2^{\varepsilon n} \sum_{k>n} \frac{1 / 2^{\varepsilon k}}{\left|1 / 2^{n}\right|^{2}} \\
& =1 / 2^{\varepsilon n} \sum_{k<n} 2^{(2-\varepsilon) k}+2^{(2-\varepsilon) n} \sum_{k>n} 1 / 2^{\varepsilon k} \asymp 2^{2(1-\varepsilon) n},
\end{aligned}
$$

which is uniformly bounded in $n$. Hence $\left(I H^{2}\right)^{\perp}$ is an $\ell^{2}$-sum of Paley-Wiener spaces (each of which possesses, for instance, the harmonic unconditional basis). In particular, picking

$$
\lambda_{n}:=r_{n} \zeta_{n}=r_{n} e^{i / 2^{n}}, \quad r_{n}=1-\frac{1}{2^{n}},
$$


the sequence $\left\{K_{n}\right\}_{n \geq 1}$, where

$$
K_{n}=\frac{k_{\lambda_{n}}^{I_{n}}}{\left\|k_{\lambda_{n}}^{I_{n}}\right\|} \in\left(I_{n} H^{2}\right)^{\perp},
$$

is an unconditional sequence in $\left(I H^{2}\right)^{\perp}$. We can introduce the family of functions

$$
f_{\beta}:=\sum_{n \geq n_{0}} \beta_{n} K_{n}
$$

where $\left\|f_{\beta}\right\|^{2} \asymp \sum_{n \geq 1}\left|\beta_{n}\right|^{2}<\infty$, and $n_{0}$ will be determined later. Let us estimate the norms $\left\|k_{\lambda_{n}}^{I_{n}}\right\|$. First observe that

$$
\alpha_{n} \frac{\lambda_{n}+\zeta_{n}}{\lambda_{n}-\zeta_{n}}=\alpha_{n} \frac{r_{n}+1}{r_{n}-1}=\frac{1}{2^{\varepsilon n}} \frac{2-1 / 2^{n}}{-1 / 2^{n}}=-\frac{2-1 / 2^{n}}{2^{(\varepsilon-1) n}} \longrightarrow 0, \quad n \rightarrow \infty .
$$

Hence

$$
\begin{aligned}
\left\|k_{\lambda_{n}}^{I_{n}}\right\|^{2} & =\frac{1-\left|I_{n}\left(\lambda_{n}\right)\right|^{2}}{1-r_{n}^{2}} \asymp \frac{1-\left|I_{n}\left(\lambda_{n}\right)\right|}{1-r_{n}}=\frac{1-\exp \left(\log \left|I_{n}\left(\lambda_{n}\right)\right|\right)}{1-r_{n}} \\
& =\frac{1-\exp \left(\alpha_{n} \frac{\lambda_{n}+\zeta_{n}}{\lambda_{n}-\zeta_{n}}\right)}{1-r_{n}} \sim \frac{1-\left(1+\alpha_{n} \frac{r_{n}+1}{r_{n}-1}\right)}{1-r_{n}} \sim \frac{2 \alpha_{n}}{\left(1-r_{n}\right)^{2}},
\end{aligned}
$$

so that

$$
\left\|k_{\lambda_{n}}^{I_{n}}\right\| \asymp \sqrt{\frac{\alpha_{n}}{\left(1-r_{n}\right)^{2}}}=\frac{\sqrt{2^{-(\varepsilon n)}}}{1 / 2^{n}}=2^{(1-\varepsilon / 2) n} .
$$

Observe now that the $\lambda_{n}$ 's belong to a Stolz domain with vertex at 1: $\Gamma_{\alpha}$ for some $\alpha>1$. Indeed,

$$
1-\left|\lambda_{n}\right|=1-r_{n}=1 / 2^{n} \simeq\left|1-\zeta_{n}\right| \asymp\left|1-\lambda_{n}\right|
$$

(this follows from (2.8)). Absorbing the equivalence constants appearing in (4.2) into $\beta=\left\{\beta_{n}\right\}_{n \geq 1} \in \ell^{2}$ with $\beta_{n} \geq 0$, and picking a suitable real number $\varphi_{0}$ (also to be determined later), we will be interested in the real part of

$$
f_{e^{i \varphi_{0} \beta}}(z)=\sum_{n \geq n_{0}} e^{i \varphi_{0}} \beta_{n} 2^{(\varepsilon-1 / 2) n} \frac{1-\overline{I_{n}\left(\lambda_{n}\right)} I_{n}(z)}{1-\overline{\lambda_{n}} z}
$$

for $z \in \Gamma_{\alpha}$. Note that when $z \in \Gamma_{\alpha}$ there exists a unique closest $\lambda_{N}$ to $z$ (in the pseudohyperbolic metric) and $\left|b_{\lambda_{N}}(z)\right| \leq \rho<1$. We have already seen that $\mathbf{R} \ni I_{n}\left(\lambda_{n}\right) \longrightarrow 1, n \rightarrow \infty$, and

$$
I_{n}\left(\lambda_{n}\right) \sim 1-\alpha_{n} \frac{1+r_{n}}{1-r_{n}} \sim 1-\frac{2}{2^{(\varepsilon-1) n}} .
$$

For $I_{n}(z)$ we need to consider

$$
\alpha_{n} \frac{z+\zeta_{n}}{z-\zeta_{n}}
$$

Note that since $\left|b_{\lambda_{N}}(z)\right| \leq \rho<1$,

$$
\left|\alpha_{n} \frac{z+\zeta_{n}}{z-\zeta_{n}}\right| \asymp\left|\alpha_{n} \frac{\lambda_{N}+\zeta_{n}}{\lambda_{N}-\zeta_{n}}\right|
$$


For $n$ and $N$ bigger than some $n_{0}$, we have $\operatorname{Re}\left(\lambda_{N}+\zeta_{n}\right) \asymp\left|\lambda_{N}+\zeta_{n}\right| \asymp 2$. We thus have to consider the denominator. We observe that by (2.8)

$$
\begin{aligned}
\left|\lambda_{N}-\zeta_{n}\right| & =\left|1-\overline{\zeta_{n}} \lambda_{N}\right| \asymp\left(1-r_{N}\right)+\left|\frac{1}{2^{n}}-\frac{1}{2^{N}}\right|=\frac{1}{2^{N}}+\left|\frac{1}{2^{n}}-\frac{1}{2^{N}}\right| \\
& \asymp \begin{cases}\frac{1}{2^{n}} & \text { if } n<N, \\
\frac{1}{2^{N}} & \text { if } n \geq N .\end{cases}
\end{aligned}
$$

As a consequence,

$$
\left|\alpha_{n} \frac{z+\zeta_{n}}{z-\zeta_{n}}\right| \asymp\left|\alpha_{n} \frac{\lambda_{N}+\zeta_{n}}{\lambda_{N}-\zeta_{n}}\right| \longrightarrow 0, \quad n, N \rightarrow \infty
$$

and

Hence

$$
I_{n}(z) \sim 1+\alpha_{n} \frac{z+\zeta_{n}}{z-\zeta_{n}}
$$

$$
\begin{aligned}
1-\overline{I_{n}\left(\lambda_{n}\right)} I_{n}(z) & \sim 1-\left(1+\alpha_{n} \frac{r_{n}+1}{r_{n}-1}\right)\left(1+\alpha_{n} \frac{z+\zeta_{n}}{z-\zeta_{n}}\right) \sim \alpha_{n} \frac{1+r_{n}}{1-r_{n}}+\alpha_{n} \frac{\zeta_{n}+z}{\zeta_{n}-z} \\
& =\alpha_{n}\left(\frac{1+r_{n}}{1-r_{n}}+\frac{\zeta_{n}+z}{\zeta_{n}-z}\right)=\alpha_{n} \frac{\left(1+r_{n}\right)\left(\zeta_{n}-z\right)+\left(1-r_{n}\right)\left(\zeta_{n}+z\right)}{\left(1-r_{n}\right)\left(\zeta_{n}-z\right)} \\
& =2 \alpha_{n} \frac{\zeta_{n}-r_{n} z}{\left(1-r_{n}\right)\left(\zeta_{n}-z\right)}=2 \alpha_{n} \zeta_{n} \frac{1-\overline{\zeta_{n}} r_{n} z}{\left(1-r_{n}\right)\left(\zeta_{n}-z\right)} \\
& =2 \alpha_{n} \zeta_{n} \frac{1-\overline{\lambda_{n}} z}{\left(1-r_{n}\right)\left(\zeta_{n}-z\right)} .
\end{aligned}
$$

From here we have

$$
\frac{1-\overline{I_{n}\left(\lambda_{n}\right)} I_{n}(z)}{1-\overline{\lambda_{n}} z} \sim \frac{2 \alpha_{n} \zeta_{n}}{\left(1-r_{n}\right)\left(\zeta_{n}-z\right)}=\frac{2}{2^{(\varepsilon-1) n}} \frac{\zeta_{n}}{\zeta_{n}-z}=\frac{2}{2^{(\varepsilon-1) n}} \frac{1-\zeta_{n} \bar{z}}{\left|\zeta_{n}-z\right|^{2}},
$$

and

$$
e^{i \varphi_{0}} \frac{1-\overline{I_{n}\left(\lambda_{n}\right)} I_{n}(z)}{1-\overline{\lambda_{n}} z}=e^{i \varphi_{0}} \frac{2}{2^{(\varepsilon-1) n}} \frac{1-\zeta_{n} \bar{z}}{\left|\zeta_{n}-z\right|^{2}}\left(1+\varepsilon_{n, z}\right),
$$

where $\varepsilon_{n, z}$ is arbitrarily small for $n, N$ sufficiently big (note that $\left|b_{\lambda_{N}}(z)\right| \leq \rho<1$ ). Since $\zeta_{n}=e^{i / 2^{n}}, n \geq n_{0}$, and $z$ is in $\Gamma_{\alpha}$, we observe that

$$
-\frac{\pi}{2} \leq \arg \left(1-\zeta_{n} \bar{z}\right) \leq \eta
$$

for some $\eta<\pi / 2$ ( $\eta$ is actually given by half of the opening angle of $\Gamma_{\alpha}$ ). Let now $\varphi_{0}=(\pi / 2-\eta) / 2$ so that

$$
-\frac{\pi}{2}<-\frac{\pi}{4}-\frac{\eta}{2} \leq \arg \left(e^{i \varphi_{0}}\left(1-\zeta_{n} \bar{z}\right)\right) \leq \frac{\pi}{4}+\frac{\eta}{2}<\frac{\pi}{2}
$$

Choosing $n_{0}$ sufficienly big, we can suppose that

$$
\left|\arg \left(1+\varepsilon_{n, z}\right)\right| \leq \frac{\varphi_{0}}{2}
$$

which implies that for $n, N \geq n_{0}$,

$$
\left|\arg \left[e^{i \varphi_{0}} \frac{1-\overline{I_{n}\left(\lambda_{n}\right)} I_{n}(z)}{1-\overline{\lambda_{n}} z}\right]\right| \leq \frac{\pi}{2}-\varphi_{0},
$$


so that taking into account (4.4)

$$
\begin{aligned}
\operatorname{Re}\left(e^{i \varphi_{0}} \frac{1-\overline{I_{n}\left(\lambda_{n}\right)} I_{n}(z)}{1-\overline{\lambda_{n}} z}\right) & \asymp\left|e^{i \varphi_{0}} \frac{1-\overline{I_{n}\left(\lambda_{n}\right)} I_{n}(z)}{1-\overline{\lambda_{n}} z}\right|=\left|\frac{1-\overline{I_{n}\left(\lambda_{n}\right)} I_{n}(z)}{1-\overline{\lambda_{n}} z}\right| \\
& \asymp \frac{2}{2^{(\varepsilon-1) n}} \frac{1}{\left|\zeta_{n}-z\right|} .
\end{aligned}
$$

For $n_{0} \leq n<N$ this expression is positive, and for $n \geq N$ (4.3) gives

$$
\left|\zeta_{n}-z\right| \asymp\left|\zeta_{n}-\lambda_{N}\right|=\left|1-\zeta_{n} \bar{\lambda}_{N}\right| \asymp \frac{1}{2^{N}} .
$$

Hence for $n \geq N$,

$$
\operatorname{Re}\left(e^{i \varphi_{0}} \frac{1-\overline{I_{n}\left(\lambda_{n}\right)} I_{n}(z)}{1-\overline{\lambda_{n}} z}\right) \asymp 2 \frac{2^{N}}{2^{(\varepsilon-1) n}},
$$

and

$$
\operatorname{Re} f_{\beta}(z) \gtrsim \sum_{n \geq N} \beta_{n} \frac{1}{2^{(1-\varepsilon / 2) n}} \frac{2^{N}}{2^{(\varepsilon-1) n}} \gtrsim 2^{N} \sum_{n \geq N} \frac{\beta_{n}}{2^{n \varepsilon / 2}} .
$$

Pick for instance $\beta_{n}=n^{-(1+\gamma) / 2}$, where $\gamma>0$ is arbitrary, so that obvioulsy $\beta_{n} \geq 0$ and $\beta \in \ell^{2}$. Then

$$
\begin{aligned}
\operatorname{Re} f_{\beta}(z) & \gtrsim 2^{N} \sum_{n \geq N} \frac{1}{n^{(1+\gamma) / 2}} \frac{1}{2^{n \varepsilon / 2}} \geq 2^{N} \frac{1}{N^{(1+\gamma) / 2}} \frac{1}{2^{N \varepsilon / 2}}=\frac{2^{(1-\varepsilon / 2) N}}{N^{(1+\gamma) / 2}} \\
& \gtrsim\left(\frac{1}{1-\left|\lambda_{N}\right|}\right)^{1-\varepsilon / 2} \frac{1}{\log ^{(1+\gamma) / 2}\left(\frac{1}{1-\left|\lambda_{N}\right|}\right)} \\
& \gtrsim\left(\frac{1}{1-|z|}\right)^{1-\varepsilon / 2} \frac{1}{\log ^{(1+\gamma) / 2}\left(\frac{1}{1-|z|}\right)}
\end{aligned}
$$

so that we loose a logarithmic term with respect to the upper estimate of the reproducing kernel (3.2).

We should mention that using the biorthogonal system to $\left(K_{n}\right)_{n}$ in the space generated by $\left(K_{n}\right)_{n}$, we could also have obtained a lower estimate, but only at the points $\lambda_{N}$, whereas in the above construction, as already mentioned in the beginning of the section, the lower estimate holds throughout the whole Stolz angle.

Finally, we point out that when $I(z) \longmapsto 1$ when $z \rightarrow 1$ in a fixed $z$ domain, it is, in general, particularly difficult to decide whether or not a sequence of reproducing kernels for $\left(I H^{2}\right)^{\perp}$, with the parameter in a Stolz domain with vertex at 1 , is an unconditional basis or not. Even when $\sup _{n}\left|I\left(\lambda_{n}\right)\right|<1$, there is a characterization known for unconditional basis which is, in general, difficult to check.

\section{References}

[AC70] Ahern, P. R., and D. N. Clark: Radial limits and invariant subspaces. - Amer. J. Math. 92, 1970, 332-342.

[AC71] Ahern, P. R., and D. N. Clark: Radial $n$th derivatives of Blaschke products. - Math. Scand. 28, 1971, 189-201.

[Coh86] Cohn, W.S.: Radial limits and star invariant subspaces of bounded mean oscillation. Amer. J. Math. 108:3, 1986, 719-749. 
[CL66] Collingwood, E. F., and A. J. Lohwater: The theory of cluster sets. - Cambridge Tracts in Mathematics and Mathematical Physics 56, Cambridge Univ. Press, Cambridge, 1966.

[Fro42] Frostman, O.: Sur les produits de Blaschke. - Kungl. Fysiografiska Sällskapets i Lund Förhandlingar 12:15, 1942, 169-182.

[HR11] Hartmann, A., and W. T. Ross: Bad boundary behavior in star invariant subspaces I. - Ark. Mat. (to appear).

[Nik86] Nikol'skil̆, N. K.: Treatise on the shift operator. - Grundlehren Math. Wiss. 273, SpringerVerlag, Berlin, 1986.

[Nik02] Nikolski, N. K.: Operators, functions, and systems: an easy reading. Volume 2. - Math. Surveys Monogr. 93, Amer. Math. Soc., Providence, RI, 2002.

Received 9 September 2011 